\title{
An ab initio and force field study on the conformation and chain flexibility of the dichlorophosphazene trimer
}

\author{
Raimund Jaeger, G. Julius Vancso* \\ University of Twente, Faculty of Chemical Technology, \\ P.O. Box 217, NL-7500 AE Enschede, The Netherlands
}

(Received: November 16, 1995; revised manuscript of January 25, 1996)

\section{SUMMARY:}

$\mathrm{Ab}$ initio molecular orbital calculations have been used to study the conformation, valence electron charge density, and chain flexibility of a dichlorophosphazene trimer $\left(\mathrm{CH}_{3}\left[\mathrm{NP}\left(\mathrm{Cl}_{2}\right)\right]_{3} \mathrm{CH}_{3}\right)$. The calculations were carried out at the restricted Hartree-Fock level with the $6-31 \mathrm{G}^{*}$ basis set. The dichlorophosphazene trimer adopts a planar transcis conformation. The valence electron charge distribution indicates strong charge separations along the backbone of the molecule, and is in agreement with Dewar's island delocalization model for bonding in linear and cyclic phosphazenes. In order to determine the height of the torsional barrier $(2,5 \mathrm{kcal} / \mathrm{mol})$, the torsional potential of a central $\mathrm{P}-\mathrm{N}$ bond of the trimer was studied with a rigid rotor scan and geometry optimizations of selected rotamers. The flexibility of the $\mathrm{P}-\mathrm{N}-\mathrm{P}$ bond angle contributes significantly to the chain flexibility. Based on the results of the ab initio calculations, an empirical force field for the dichlorophosphazene trimer was developed. The energy expression includes bond stretch, angle bend, electrostatic, van der Waals, and torsional potential terms. A relaxed scan with the force field achieves good agreement with the ab initio results for the torsional potential in the vicinity of the stable conformation, and an excellent agreement with the ab initio results on changes in the $\mathrm{P}^{2} \mathrm{~N}^{2} \mathrm{P}^{3}$ bond angle and the $\mathrm{N}^{1} \mathrm{P}^{2}-\mathrm{N}^{2} \mathrm{P}^{3}$ dihedral angle during a full rotation around the $\mathrm{N}^{2}-\mathrm{P}^{3}$ bond.

\section{Introduction}

Recently, interest in the design of novel, high value added materials with specific chemical or physical characteristics has been increasing. Most of the commercially used polymeric materials are carbon-based. In searching for novel polymers, it is therefore important to investigate the synthesis and physical properties of macromolecules which have inorganic elements in the main chain in order to evaluate their potential for technological applications.

Compared to organic polymers, inorganic macromolecules have interesting and unusual chemical, mechanical, electronic, and/or thermal properties. The unique properties of polymeric materials (like the large reversible deformability of elastomers, or the high impact strength of thermoplastics) are due to the variety of different conformations which the polymer chains can adopt ${ }^{1)}$. Knowledge of the stable conformation and the flexibility of the polymer main chain (i.e., the energy barriers between different conformations) is therefore essential to understand the physical properties of polymers. A molecular geometry which differs from the geometry of a carbon-based backbone (e.g., larger bond lengths and bond angles), and a highly flexible main chain cause the exceptional properties of some inorganic polymers ${ }^{2}$. 
The glass transition temperature of a polymer is influenced by the chain flexibility $^{3-5)}$. For example, the flexibility of the poly(dimethylsiloxane) ([Si( $\left.\left.\mathrm{CH}_{3}\right)-\mathrm{O}\right]_{n}$ ) backbone leads to an extraordinarily low glass transition temperature $T_{\mathrm{g}}=-126^{\circ} \mathrm{C}$. Low glass transition temperatures of some polyphosphazenes $\left(\left[\mathrm{PR}_{2}-\mathrm{N}\right]_{n}\right)$ are also attributed to the high torsional mobility of the phosphazene backbone.

Computational techniques have recently become more important for studying polymer structures and properties ${ }^{6,7)}$. In this paper we present a computational study of the structure and chain flexibility of a trimer of poly(dichlorophosphazene) (PDCP), $\left(\mathrm{PCl}_{2}\right)_{n}$ (see Figs. 1 and 2).

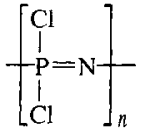

1

Fig. 1. Chemical repeat unit of the poly(dichlorophosphazene) polymer. The crystallographic repeat unit consists of two monomers. Single and double $\mathrm{P}-\mathrm{N}$ bonds are in terms of their length and electronic structure - unlike $\mathrm{C}-\mathrm{C}$ single and double bonds - almost identical<smiles>C[N+]=[P-](Cl)(Cl)N=[P+](Cl)(Cl)N=P(C)(Cl)Cl</smiles>

2
Fig. 2. Chemical structure of the dichlorophosphazene trimer

The synthesis of phosphazene polymers was pioneered by Allcock and coworkers. $\mathrm{Ab}$ initio molecular orbital calculations on the structure and bonding of cyclic and short chain linear phosphazene molecules have been carried out by Trinquier ${ }^{8,9}$ and Ferris et al. ${ }^{10-12)}$. Several authors reported on the development of empirical force fields for phosphazene molecules ${ }^{13-16)}$. The parameterization of these force fields was based on experimental data.

We use ab initio molecular orbital calculations in order to study the structure, torsional potentials, charge density distributions, and force constants of the dichlorophosphazene trimer which is used as a short chain analogue of PDCP. Based on the ab initio results, we develop and parameterize an empirical force field for the dichlorophosphazene trimer which simulates the flexibility of the phosphazene main chain.

\section{Method of computation}

We used a HP 755 workstation and an Indigo SGI RS 4000 workstation in order to perform the Gaussian 92 ab initio molecular orbital calculations ${ }^{17,18)}$. The calculations were carried out at the restricted Hartree-Fock (RHF) level of theory. We used the $6-31 \mathrm{G}^{*}$ basis set for the Gaussian orbital wave functions. The program employs a gradient method for geometry optimizations.

The graphic representations of the molecules were created with the CERIUS molecular modeling software package ${ }^{19)}$ and the IRIS Explorer graphics package. 
We developed a Fortran 77 routine for the force field calculations. The program uses a conjugate-directions algorithm for the geometry optimizations which was written by R. P. Brent. The routine was obtained through the internet by the 'Guide to Available mathematical Software' which is maintained by the National Institute of Standards and Technology.

\section{Ab initio results and discussion}

\section{Conformation and charge density distribution of the dichlorophosphazene trimer}

The molecular geometry of the dichlorophosphazene trimer (see Fig. 2) was restricted to a conformation with $\mathrm{C}_{\mathrm{s}}$ symmetry for the geometry optimizations. The mirror plane contained the backbone atoms (i.e., the phosphorus, nitrogen, and carbon atoms). We carried out a frequency calculation on the molecular geometry which resulted from the geometry optimization in order to verify that the optimized geometry corresponds to a minimum and not to a transition state of the potential energy surface (PES). The dichlorophosphazene trimer adopts a planar trans-cis conformation (see Fig. 3). The bond lengths, bond angles, and dihedral angles of the stable conformation are shown in Tab. 1. We observe a slight alternation in $\mathrm{P}-\mathrm{N}$ bond lengths (up to $0,1 \AA$ ). The longer, 'single' $\mathrm{P}-\mathrm{N}$ bonds are parallel to the direction of the main chain, and the shorter, 'double' $\mathrm{P}=\mathrm{N}$ bonds form an angle with the direction of the main chain. The energy of the trans-cis conformation of the dichlorophosphazene trimer with respect to the quantum chemical standard state (i.e., the state where all electrons and nuclei are infinitely separated) is $E\left(\mathrm{RHF} / 6-31 \mathrm{G}^{*}\right)=$ $-2523680,9 \mathrm{kcal} / \mathrm{mol}$.

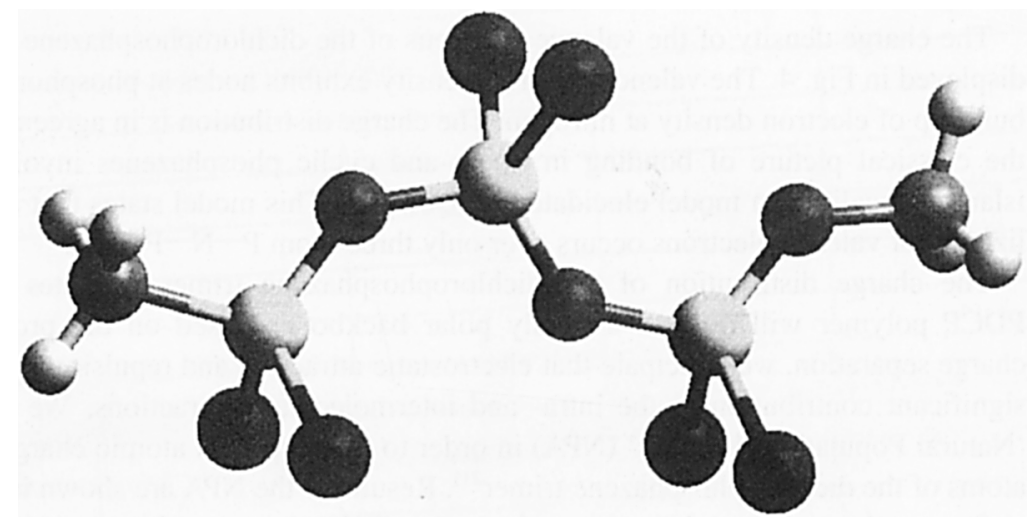

Fig. 3. 'Ball and stick' model of the stable conformation of the dichlorophosphazene trimer 
Tab. 1. Molecular geometry of the dichlorophosphazene trimer. The dihedral angles which are marked with the symbol * were kept fixed during the geometry optimization

\begin{tabular}{llll}
\hline \multicolumn{4}{c}{ Bond lengths in $\AA$} \\
\hline $\mathrm{P}^{1}-\mathrm{C}^{1}$ & 1,7971 & $\mathrm{Cl}^{1}-\mathrm{P}^{1}$ & \\
$\mathrm{~N}^{1}-\mathrm{P}^{1}$ & 1,5369 & $\mathrm{Cl}^{3}-\mathrm{P}^{2}$ & 2,0075 \\
$\mathrm{P}^{2}-\mathrm{N}^{1}$ & 1,5995 & $\mathrm{Cl}^{5}-\mathrm{P}^{3}$ & 2,0097 \\
$\mathrm{~N}^{2}-\mathrm{P}^{2}$ & 1,5376 & $\mathrm{H}^{1}-\mathrm{C}^{\mathrm{I}}$ & 2,0514 \\
$\mathrm{P}^{3}-\mathrm{N}^{2}$ & 1,6034 & $\mathrm{H}^{2}-\mathrm{C}^{1}$ & 1,0815 \\
$\mathrm{~N}^{3}-\mathrm{P}^{3}$ & 1,5079 & $\mathrm{H}^{4}-\mathrm{C}^{2}$ & 1,0829 \\
$\mathrm{C}^{2}-\mathrm{N}^{3}$ & 1,4396 & $\mathrm{H}^{5}-\mathrm{C}^{2}$ & 1,0832 \\
\hline \multicolumn{4}{c}{ Bond angles in degree } \\
\hline$\angle \mathrm{N}^{1} \mathrm{P}^{1} \mathrm{C}^{1}$ & 110,81 & $\angle \mathrm{Cl}^{3} \mathrm{P}^{2} \mathrm{~N}^{2}$ & 1,0877 \\
$\angle \mathrm{P}^{2} \mathrm{~N}^{1} \mathrm{P}^{1}$ & 135,34 & $\angle \mathrm{Cl}^{5} \mathrm{P}^{3} \mathrm{~N}^{3}$ & 114,54 \\
$\angle \mathrm{N}^{2} \mathrm{P}^{2} \mathrm{~N}^{1}$ & 113,37 & $\angle \mathrm{H}^{1} \mathrm{C}^{1} \mathrm{P}^{1}$ & 115,74 \\
$\angle \mathrm{P}^{3} \mathrm{~N}^{2} \mathrm{P}^{2}$ & 134,31 & $\angle \mathrm{H}^{2} \mathrm{C}^{1} \mathrm{P}^{1}$ & 110,83 \\
$\angle \mathrm{N}^{3} \mathrm{P}^{3} \mathrm{~N}^{2}$ & 115,97 & $\angle \mathrm{H}^{4} \mathrm{C}^{2} \mathrm{~N}^{3}$ & 108,30 \\
$\angle \mathrm{C}^{2} \mathrm{~N}^{3} \mathrm{P}^{3}$ & 131,61 & $\angle \mathrm{H}^{5} \mathrm{C}^{2} \mathrm{~N}^{3}$ & 108,90 \\
$\angle \mathrm{Cl}^{1} \mathrm{P}^{1} \mathrm{~N}^{1}$ & 115,19 & & 112,24 \\
\hline
\end{tabular}

Dihedral angles in degree

\begin{tabular}{lrrr}
\hline$\angle \mathrm{Cl}^{1} \mathrm{P}^{1}-\mathrm{N}^{1} \mathrm{C}^{1}$ & 119,6 & $\angle \mathrm{N}^{2} \mathrm{P}^{2}-\mathrm{N}^{1} \mathrm{P}^{1}$ & $0,0^{*}$ \\
$\angle \mathrm{Cl}^{3} \mathrm{P}^{2}-\mathrm{N}^{2} \mathrm{~N}^{1}$ & 120,8 & $\angle \mathrm{P}^{3} \mathrm{~N}^{2}-\mathrm{P}^{2} \mathrm{~N}^{1}$ & $180,0^{*}$ \\
$\angle \mathrm{Cl}^{5} \mathrm{P}^{3}-\mathrm{N}^{3} \mathrm{~N}^{2}$ & $-122,2$ & $\angle \mathrm{N}^{3} \mathrm{P}^{3}-\mathrm{N}^{2} \mathrm{P}^{2}$ & $0,0^{*}$ \\
$\angle \mathrm{H}^{2} \mathrm{C}^{1}-\mathrm{P}^{1} \mathrm{H}^{1}$ & 120,9 & $\angle \mathrm{C}^{2} \mathrm{~N}^{3}-\mathrm{P}^{3} \mathrm{~N}^{2}$ & $180,0^{*}$ \\
$\angle \mathrm{H}^{5} \mathrm{C}^{2}-\mathrm{N}^{3} \mathrm{H}^{3}$ & 119,1 & $\angle \mathrm{H}^{1} \mathrm{C}^{1}-\mathrm{P}^{1} \mathrm{~N}^{1}$ & $180,0^{*}$ \\
$\angle \mathrm{P}^{2} \mathrm{~N}^{1}-\mathrm{P}^{1} \mathrm{C}^{1}$ & $180,0^{*}$ & $\angle \mathrm{H}^{4} \mathrm{C}^{2}-\mathrm{N}^{3} \mathrm{P}^{3}$ & $180,0^{*}$
\end{tabular}

The charge density of the valence electrons of the dichlorophosphazene trimer is displayed in Fig. 4. The valence electron density exhibits nodes at phosphorus, and a build-up of electron density at nitrogen. The charge distribution is in agreement with the classical picture of bonding in linear and cyclic phosphazenes involving the island delocalization model elucidated by Dewar ${ }^{20)}$. This model states that a delocalization of valence electrons occurs over only three-atom $\mathrm{P}-\mathrm{N}-\mathrm{P}$ units.

The charge distribution of the dichlorophosphazene trimer indicates that the PDCP polymer will possess a highly polar backbone. Based on the pronounced charge separation, we anticipate that electrostatic attraction and repulsion will give a significant contribution to the intra- and intermolecular interactions. We used the 'Natural Population Analysis' (NPA) in order to assign partial atomic charges to the atoms of the dichlorophosphazene trimer ${ }^{21)}$. Results of the NPA are shown in Tab. 2.

Our results on the stable geometry, the bond length alternation, and the charge distribution are in agreement with the results of Ferris and co-workers ${ }^{10-12)}$. Ferris et al. carried out a detailed ab initio study on the bond structure and bond lengths alter- 
Fig. 4. Charge density of the valence electrons of the dichlorophosphazene trimer. The surface denotes the volume which includes $85 \%$ of the charge density of the valence electrons

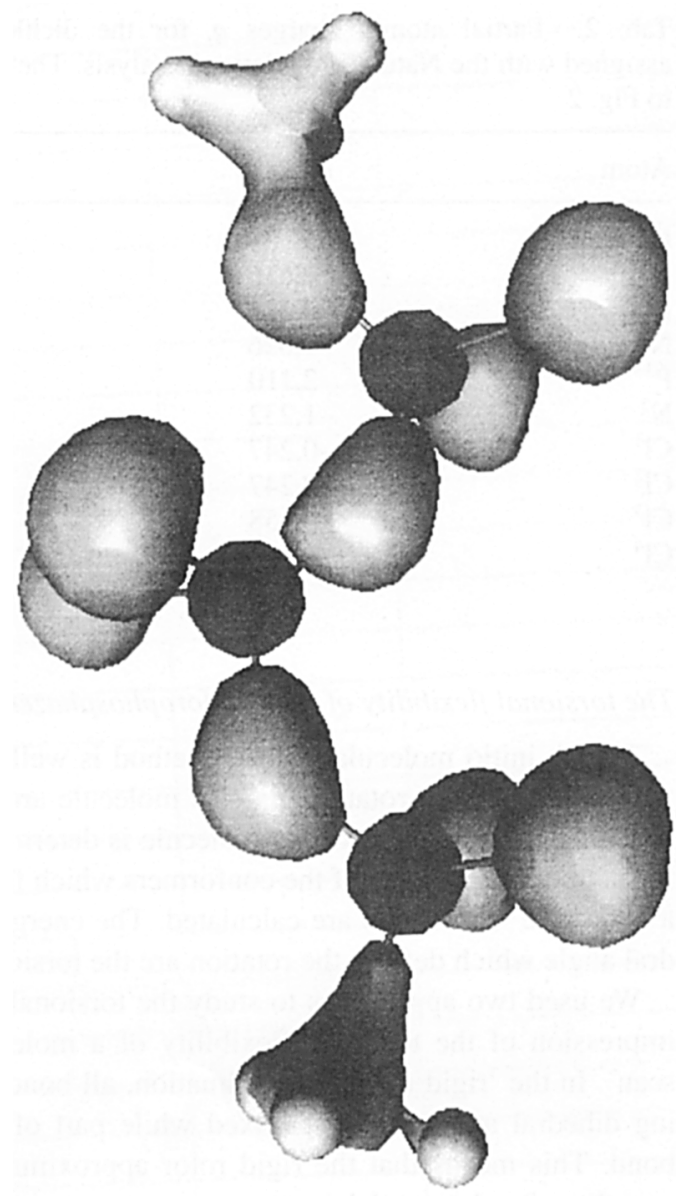

nations of small cyclic and linear phosphazene molecules with hydrogen and fluorine as substituents bonded to phosphorus. Based on the results for bond length alternations of a series of linear phosphazene molecules with increasing molecular weight, Ferris and co-workers suggest bond lengths which alternate by $0,05 \AA$ for the polymer. Although chemical bonding in linear phosphazene backbones cannot be interpreted in terms of single and double bonds, small bond length alternations can have a significant influence on the vibrational and electronic properties of polymers (consider, e.g., bond length alternations in polyenes ${ }^{21}$ ). However, the electronic structure of phosphazenes differs from the electronic structure of a potential conductor such as polyacetylene. The smallest possible repeat unit of PDCP consists of a $\mathrm{PCl}_{2}$ moiety and a nitrogen atom. The phosphorus and the nitrogen atom each contribute one electron to the highest occupied band, and this band is therefore filled. A Peierls distortion (like in polyacetylene) will not occur, since a half-filled conducting band is required for this phenomenon. 
Tab. 2. Partial atomic charges $q_{i}$ for the dichlorophosphazene trimer which were assigned with the Natural Population Analysis. The numbering of the atoms corresponds to Fig. 2

\begin{tabular}{lrlr}
\hline Atom & \multicolumn{1}{c}{ in $e$} & Atom & $q$ in $e$ \\
\hline $\mathrm{P}^{\mathrm{l}}$ & 1,913 & $\mathrm{Cl}^{5}$ & $-0,340$ \\
$\mathrm{~N}^{1}$ & $-1,610$ & $\mathrm{Cl}^{6}$ & $-0,340$ \\
$\mathrm{P}^{2}$ & 2,135 & $\mathrm{C}^{1}$ & $-1,042$ \\
$\mathrm{~N}^{2}$ & $-1,626$ & $\mathrm{C}^{2}$ & $-0,392$ \\
$\mathrm{P}^{3}$ & 2,110 & $\mathrm{H}^{1}$ & 0,271 \\
$\mathrm{~N}^{3}$ & $-1,232$ & $\mathrm{H}^{2}$ & 0,280 \\
$\mathrm{Cl}^{1}$ & $-0,247$ & $\mathrm{H}^{3}$ & 0,280 \\
$\mathrm{Cl}^{2}$ & $-0,247$ & $\mathrm{H}^{4}$ & 0,212 \\
$\mathrm{Cl}^{3}$ & $-0,258$ & $\mathrm{H}^{5}$ & 0,195 \\
$\mathrm{Cl}^{4}$ & $-0,258$ & $\mathrm{H}^{6}$ & 0,195
\end{tabular}

\section{The torsional flexibility of the dichlorophosphazene trimer}

The ab initio molecular orbital method is well-suited for determining the energy which is needed to rotate parts of a molecule around a given bond. As a first step, the stable conformation of the molecule is determined. Starting from the stable conformation, the energies of the conformers which form during a given rotation around a bond (the 'rotamers') are calculated. The energy values as a function of the dihedral angle which defines the rotation are the torsional potential.

We used two approaches to study the torsional potentials. In order to gain a first impression of the torsional flexibility of a molecule, we performed a 'rigid rotor scan'. In the 'rigid rotor' approximation, all bond lengths, bond angles and remaining dihedral angles are kept fixed while part of the molecule is rotated around a bond. This means that the rigid rotor approximation yields an upper limit for the actual torsional potential.

In addition, we compared the results of the rigid rotor scan with results of a 'fully optimized' or 'relaxed' scan. In the 'relaxed' scan the energies of fully optimized structures which form during a rotation are determined. Therefore, the relaxed scan will yield shapes of torsional potentials which are close to those in reality.

Compared to the relaxed scan, the rigid rotor scan is significantly less time-consuming, since geometry optimizations are omitted while one part of the molecule is rotated around a bond. The rigid rotor approximation is not successful in determining absolute barrier heights and shapes of torsional potentials, since adjustments of the molecular geometry which reduce strains (e.g., bending of bond angles or rotation of side groups) during the rotation of a part of the molecule are not considered. A comparison of the result of a rigid rotor scan and the energies of the geometry optimized rotamers (relaxed scan) will show the contribution of the flexibility of the bond angles to the torsional mobility of the PDCP backbone.

The $\mathrm{P}^{2} \mathrm{~N}^{2}-\mathrm{P}^{3} \mathrm{~N}^{3}$ dihedral angle of the dichlorophosphazene trimer was rotated from $0^{\circ}$ to $180^{\circ}$ in $15^{\circ}$ steps during a rigid rotor scan - a full rotation was not neces- 
sary owing to the $\mathrm{C}_{\mathrm{s}}$ symmetry of the molecule. In addition, we carried out geometry optimizations for selected rotamers which form during a rotation around the $\mathrm{N}^{2}-\mathrm{P}^{3}$ bond. The results of the rigid-rotor and the relaxed scan are shown in Fig. 5.

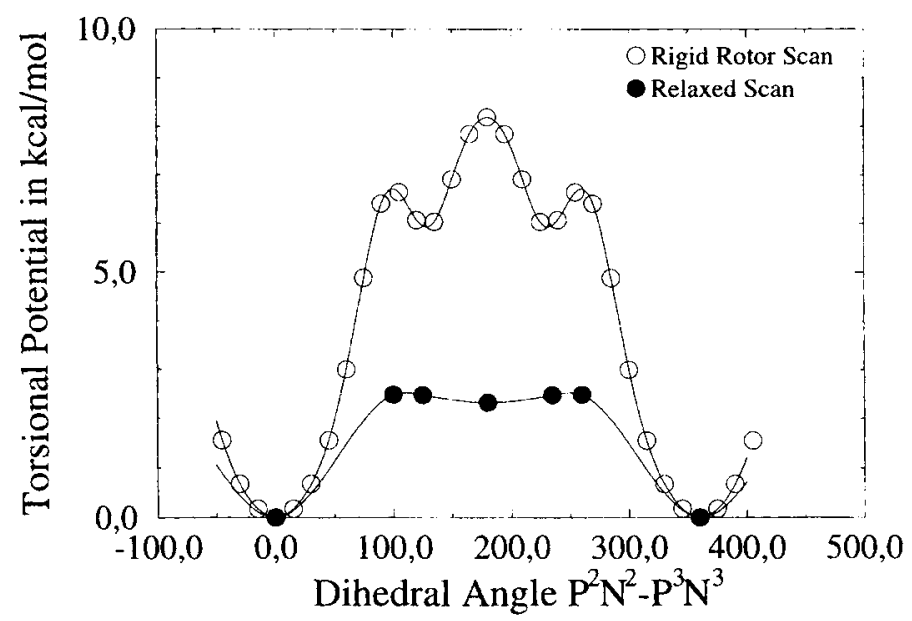

Fig. 5. Torsional potential of the $\mathrm{N}^{2}-\mathrm{P}^{3}$ bond (angle in degree). Open symbols denote results of rigid-rotor scans; filled symbols denote results of relaxed scans. The torsional angle $\phi=0^{\circ}$ corresponds to the cis conformation

The torsional potential which was obtained with the rigid rotor approximation exhibits three maxima. The local maxima at $\angle \mathrm{P}^{2} \mathrm{~N}^{2}-\mathrm{P}^{3} \mathrm{~N}^{3}=100^{\circ}$ and $260^{\circ}$ occur at conformations with minimal distance between one chlorine atom bonded to phosphorus atom $\mathrm{P}^{2}$ and another chlorine atom bonded to phosphorus atom $\mathrm{P}^{3}$. The global maximum at $\angle \mathrm{P}^{2} \mathrm{~N}^{2}-\mathrm{P}^{3} \mathrm{~N}^{3}=180^{\circ}$ corresponds to the eclipsed conformation.

The height of the torsional barrier which resulted from the relaxed scan $(2,5 \mathrm{kcal} /$ $\mathrm{mol}$ ) is significantly lower than the barrier which was obtained with the rigid rotor approximation $(8,2 \mathrm{kcal} / \mathrm{mol})$. The $\mathrm{P}^{2} \mathrm{~N}^{2} \mathrm{P}^{3}$ bond angle undergoes a significant variation during a full rotation (see Fig. 8). The bond angle opens up to $24,0^{\circ}$. The widening of the $\mathrm{P}^{2} \mathrm{~N}^{2} \mathrm{P}^{3}$ bond angle decreases the repulsive interaction between the $\mathrm{PCl}_{2}$ moieties, and therefore lowers the torsional barrier. The flexibility of the $\mathrm{P}-\mathrm{N}-\mathrm{P}$ bond angles contributes therefore significantly to the flexibility of the phosphazene backbone.

We want to discuss two results of our previous ab initio molecular orbital study on the structure and chain flexibility of halogenated poly(thionylphosphazene)s $\left[(\mathrm{NSOX})\left(\mathrm{NPCl}_{2}\right)_{2}\right]_{n}, \mathrm{X}=\mathrm{F}$ or $\mathrm{Cl}$, which are of significance for the present study on the chain flexibility of $\mathrm{PDCP}^{23)}$. In this work it was concluded that electron correlation has a negligible influence on the height of the torsional barriers of halogenated poly(thionylphosphazene)s (electron correlation reduces the barrier by $3 \%$ ). In addition, the torsional flexibility of a bond of the main chain is governed by its immedi- 
ate 'molecular environment'. The torsional potential for a rotation around a $\mathrm{P}-\mathrm{N}$ bond of the 'phosphazene end' of the poly(thionylphosphazene) monomer unit differs at most by $5 \%$ from the torsional potential of a central $\mathrm{P}-\mathrm{N}$ bond in the dichlorophosphazene trimer. This observation justifies the significance of the calculations on low molecular weight analogues for the polymeric system.

\section{An empirical force field for the dichlorophosphazene trimer}

The ab initio molecular orbital technique, which was used in the previous section, requires time-consuming calculations. Ab initio calculations on 'larger' molecules (e.g. macromolecules or molecules with larger side groups) are currently not feasible, since the required computer time increases sharply with the number of atomic orbitals which are used to carry out the calculations ${ }^{24)}$. A possible solution to this problem is to use 'classical mechanical' or 'empirical' force field calculations for larger molecules ${ }^{7,25)}$. Force field calculations approximate the potential energy surface (PES) $E(\boldsymbol{r}, \boldsymbol{\theta}, \boldsymbol{\varphi})$ with an analytical expression (the 'energy expression') which is composed of a sum of energy terms. $r=\left(r_{2}, \ldots, r_{N}\right)$ denotes the bond-lengths, $\theta=$ $\left(\vartheta_{3}, \ldots, \vartheta_{N}\right)$ the bond-angles, and $\varphi=\left(\varphi_{4}, \ldots, \varphi_{N}\right)$ the dihedral angles which are necessary to define the molecular geometry. The various energy terms represent changes in the energy of the molecule which are caused by particular internal motions or interactions ${ }^{26,27)}$. Previous results (e.g. experimental and/or ab initio results) are absolutely necessary for a parameterization of the various energy term of the energy expression.

\section{Energy expression and parameterization}

We tried to develop an empirical force field which simulates the flexibility of the PDCP main chain. We used the ab initio results on the dichlorophosphazene trimer (molecular structure, partial atomic charges, torsional barriers, and force constants for bond stretch and angle bend motions) in order to parameterize and test the energy expression of the force field. The energy expression includes harmonic terms for bond stretch (1) and angle bend (2) motions, electrostatic (3) and van der Waals (4) potentials for the non-bonded intramolecular interaction, and torsional potential terms (5) for selected torsional motions:

$$
\begin{aligned}
E(\boldsymbol{r}, \boldsymbol{\theta}, \boldsymbol{\varphi})= & \sum_{i=2}^{N} \frac{1}{2} k_{i}^{\mathrm{s}}\left(r_{i}-r_{i}^{0}\right)^{2} \\
& +\sum_{i=3}^{N} \frac{1}{2} k_{i}^{\mathrm{b}}\left(\theta_{i}-\theta_{i}^{0}\right)^{2} \\
& +\frac{\alpha}{4 \pi \varepsilon_{0}} \sum_{i<j}, \frac{q_{i} q_{j}}{r_{i j}}
\end{aligned}
$$




$$
\begin{aligned}
& +\sum_{i<j} D_{i j}^{0}\left[\left(\frac{r_{i j}^{0}}{r_{i j}}\right)^{12}-2\left(\frac{r_{i j}^{0}}{r_{i j}}\right)^{6}\right] \\
& +\sum_{i=4,6,8} k^{\prime}\left(1-\cos \left(2 \varphi_{i}\right)\right)
\end{aligned}
$$

$r_{i}^{0}$ denotes the 'zero-strain' values for bond lengths, $k_{i}^{\mathrm{s}}$ the force constant for bond stretching, $\theta_{i}^{0}$ the zero-strain values for bond angles, and $k_{i}^{\mathrm{b}}$ the force constant for bond angle bending. $\alpha$ is an adjustable parameter, $\varepsilon_{0}$ denotes the permittivity of the vacuum, $w_{i}$ are NPA partial atomic charges, and $r_{i j}$ are the inter-nuclear distances. $r_{i j}^{0}$ denotes the inter-nuclear distance at which the Lennard-Jones potential reaches its minimum value $D_{i j}^{0}$. Pairs of atoms which are covalently bonded or which form a bond form a bond angle with a third covalently bonded atom are not included in the summation $\sum_{i<j}^{\prime}$ in the energy terms of non-bonded interactions. The contribution of covalently bonded atoms to the non-bonded interaction will be included in the 'bond-stretch' terms, and the contribution of two atoms which form a bond angle with a third atom is included in the 'angle-bend' energy term. The torsional potential terms (5) restrict the mobility of torsional motion around the dihedral angles $\varphi_{4}=$ $\angle \mathrm{C}^{1} \mathrm{P}^{1}-\mathrm{N}^{1} \mathrm{P}^{2}, \varphi_{6}=\angle \mathrm{N}^{1} \mathrm{P}^{2}-\mathrm{N}^{2} \mathrm{P}^{3}$, and $\varphi_{8}=\angle \mathrm{C}^{2} \mathrm{~N}^{3}-\mathrm{P}^{3} \mathrm{~N}^{2}$

We used the parameters $D_{i j}^{0}$ and $r_{i j}^{0}$ of the Lennard-Jones 12-6 potential which are implemented in the Dreiding II force field ${ }^{28)}$ (see Tab. 3). The geometric means $D_{\mathrm{A}, \mathrm{B}}^{0}=\sqrt{D_{\mathrm{A}, \mathrm{A}}^{0} D_{\mathrm{B}, \mathrm{B}}^{0}}$ and $r_{\mathrm{A}, \mathrm{B}}^{0}=\sqrt{r_{\mathrm{A}, \mathrm{A}}^{0} r_{\mathrm{B}, \mathrm{B}}^{0}}$ of the parameters for like atoms were used for the parameters of pairs of unlike atoms.

Tab. 3. Parameters $r^{0}$ and $D^{0}$ for the Lennard-Jones 12-6 potential

\begin{tabular}{lll}
\hline Atom & $r^{0}$ in $\AA$ & $D_{0}$ in $\mathrm{kcal} / \mathrm{mol}$ \\
\hline $\mathbf{P}$ & 4,15 & 0,32 \\
$\mathrm{~N}$ & 3,66 & 0,077 \\
$\mathrm{Cl}$ & 3,95 & 0,283 \\
$\mathrm{C}$ & 4,15 & 0,25 \\
$\mathrm{H}$ & 3,20 & 0,015
\end{tabular}

The atomic centered charges $w_{i}$ were based on partial atomic charges, which were assigned with the Natural Population Analysis (see Tab. 2). A common scaling factor $\sqrt{\alpha}$ was applied to all charges since the magnitude of partial atomic charges depends on the population analysis scheme ${ }^{29}$. We used a non-linear regression of a rigid rotor scan around the $\mathrm{P}^{2}-\mathrm{N}^{3}$ bond in order to determine the scaling factor $\alpha=$ 0,348 (see Fig. 6).

$\mathrm{Ab}$ initio RHF/6-31G* geometry optimizations and frequency calculations were used in order to determine the constants $k_{i}^{\mathrm{s}}$ and $k_{i}^{\mathrm{b}}$ and the zero-strain bond lengths $r_{i}^{0}$ and bond angles $\theta_{i}^{0}$. 


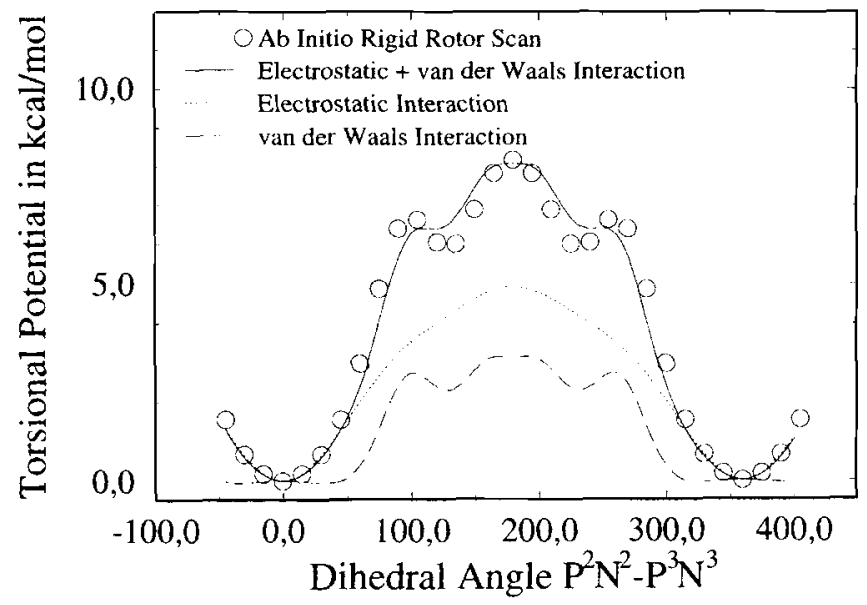

Fig. 6. Non-linear fit of an ab initio rigid rotor scan around the $\mathrm{P}^{2}-\mathrm{N}^{3}$ bond (angle in degree) with electrostatic and van der Waals potentials. The scaling factor $\alpha$ of the electrostatic term is an adjustable parameter

The geometry optimization yields a stationary point of the PES, i.e.

$$
\frac{\partial E}{\partial r_{i}}=0 \quad \frac{\partial E}{\partial \theta_{j}}=0 \quad \frac{\partial E}{\partial \varphi_{l}}=0
$$

and the frequency calculation determines the second derivatives of the potential energy surface at the stationary point:

$$
\frac{\partial^{2} E}{\partial r_{i}^{2}}=H_{i i} \quad \frac{\partial^{2} E}{\partial \theta_{j}^{2}}=H_{j j} \quad \frac{\partial^{2} E}{\partial \varphi_{l}^{2}}=H_{l l} \quad \frac{\partial^{2} E}{\partial r_{i} \theta_{j}}=H_{i j}
$$

The ab initio results include contributions from non-bonded interactions, which are accounted for separately in the force field calculation. Therefore, the influence of the non-bonded interactions on the geometry optimized bond lengths $r_{i}$ and bond angles $\theta_{i}$, and on the elements $H_{i j}$ of the matrix of second derivatives of the PES has to be removed in order to obtain the zero-strain values $r_{i}^{0}, \theta_{j}^{0}, k_{i}^{\mathrm{s}}, k_{j}^{\mathrm{b}}$

$$
\begin{aligned}
& \frac{\partial E}{\partial r_{i}}=0=k_{i}^{\mathrm{s}}\left(r_{i}-r_{i}^{0}\right)+\frac{\partial}{\partial r_{i}}\left[E_{\mathrm{estat}}+E_{\mathrm{vdW}}\right] \\
& \frac{\partial E}{\partial \theta_{j}}=0=k_{j}^{\mathrm{b}}\left(\theta_{j}-\theta_{j}^{0}\right)+\frac{\partial}{\partial \theta_{j}}\left[E_{\text {estat }}+E_{\mathrm{vdW}}\right]
\end{aligned}
$$




$$
\begin{gathered}
\frac{\partial^{2} E}{\partial r_{i}^{2}}=H_{i i}=k_{i}^{\mathrm{s}}+\frac{\partial^{2}}{\partial r_{i}^{2}}\left[E_{\text {estat }}+E_{\mathrm{vdW}}\right] \\
\frac{\partial^{2} E}{\partial \theta_{j}^{2}}=H_{j j}=k_{j}^{\mathrm{b}}+\frac{\partial^{2}}{\partial \theta_{j}^{2}}\left[E_{\text {estat }}+E_{\mathrm{vdW}}\right] \\
\Longrightarrow k_{i}^{\mathrm{s}}=H_{i i}-\frac{\partial^{2}}{\partial r_{i}^{2}}\left[E_{\mathrm{estat}}+E_{\mathrm{vdw}}\right] \quad r_{i}^{0}=r_{i}-\frac{\frac{\partial}{\partial r_{i}}\left[E_{\mathrm{estat}}+E_{\mathrm{vdW}}\right]}{H_{i i}-\frac{\partial^{2}}{\partial r_{i}^{2}}\left[E_{\mathrm{estat}}+E_{\mathrm{vdW}}\right]} \\
\Longrightarrow k_{j}^{\mathrm{b}}=H_{j j}-\frac{\partial^{2}}{\partial \theta_{j}^{2}}\left[E_{\mathrm{estat}}+E_{\mathrm{vdW}}\right] \quad \theta_{j}^{0}=\theta_{j}-\frac{\frac{\partial}{\partial \theta_{j}}\left[E_{\mathrm{estat}}+E_{\mathrm{vdW}}\right]}{H_{j j}-\frac{\partial^{2}}{\partial \theta_{j}^{2}}\left[E_{\mathrm{estat}}+E_{\mathrm{vdW}}\right]}
\end{gathered}
$$

where $E_{\text {estat }}$ refers to term (3), and $E_{\mathrm{vdw}}$ refers to term (4) of the energy expression.

The geometry optimized bond length $r_{i}$ and bond angles $\theta_{j}$, the second derivatives of the PES at the optimized geometry $H_{j j}$, the zero-strain bond length $r_{i}^{0}$ and zerostrain bond angles $\theta_{j}^{0}$, and the constants $k_{i}^{\mathrm{s}}$ and $k_{j}^{\mathrm{b}}$ are shown in Tabs. 4 and 5 . The values which are shown in the tables were calculated with the constants for the van der Waals interaction, the partial charges, and the scaling constant $\alpha$, which were discussed above.

The harmonic approximation for the bond-stretch and angle-bend potentials yielded satisfactory results for small variations of bond lengths and bond angles. However, the $\mathrm{P}^{2} \mathrm{~N}^{2} \mathrm{P}^{3}$ bond angle undergoes large changes $\left(24^{\circ}\right)$ during a torsion around the $\mathrm{N}^{2}-\mathrm{P}^{3}$ bond. We therefore adjusted the values of $H_{j j}$ for the $\mathrm{P}-\mathrm{N}-\mathrm{P}$ bond angle bending motion to $0,05 \mathrm{kcal} \cdot \mathrm{mol}^{-1} \cdot$ degree $^{-2}$.

Geometry optimizations with an energy expression which includes only the terms (1)-(4) (i.e., without the torsional potential terms (5)) show that the force field is able to capture certain characteristics of the PES. The force field determines the planar trans-cis conformation as a minimum of the PES in an unconstrained geometry optimization, and a geometry optimization of the eclipsed conformation (which corresponds to a $\mathrm{P}^{2} \mathrm{~N}^{2}-\mathrm{P}^{3} \mathrm{~N}^{3}$ dihedral angle of $180^{\circ}$ ) yields values of the torsional barrier and the $\mathrm{P}^{2} \mathrm{~N}^{2} \mathrm{P}^{3}$ bond angle bend which are close to those determined with an $a b$ initio relaxed scan - provided that the molecule is restricted to the planar (i.e., it is restricted to adopt the $\mathrm{C}_{\mathrm{s}}$ symmetry).

If an unconstrained geometry optimization is carried out starting with an initial guess which is 'far away' from the trans-cis conformation (e.g. an all-trans conformation), the force field finds a gauche conformation as ground state conformation $\left(\angle \mathrm{P}^{2} \mathrm{~N}^{1}-\mathrm{P}^{1} \mathrm{C}^{1}=-63,6^{\circ}\right.$ instead of $\left.180.0^{\circ}\right)$. The planar trans-cis conformation is a 
Tab. 4. Geometry optimized bond lengths $r_{i}$, zero-strain bond lengths $r_{i}^{0}$, second derivatives of the PES $H_{i i}$, and constants $k_{i}^{s}$ for the dichlorophosphazene trimer

\begin{tabular}{llllll}
\hline Bond & $r_{i}$ & $r_{i}^{0}$ & & $H_{i i}$ & $k_{i}^{\mathrm{s}}$ \\
\cline { 2 - 3 } \cline { 5 - 6 } & & in $\AA$ & & & \multicolumn{2}{c}{ in $\mathrm{kcal} \cdot \mathrm{mol}^{-1} \cdot \AA^{-2}$} \\
\hline $\mathrm{C}^{1}-\mathrm{P}^{1}$ & 1,797 & 1,905 & 158,356 & 154,377 \\
$\mathrm{P}^{1}-\mathrm{N}^{1}$ & 1,537 & 1,637 & & 370,342 & 366,750 \\
$\mathrm{~N}^{1}-\mathrm{P}^{2}$ & 1,600 & 1,740 & & 273,965 & 278,549 \\
$\mathrm{P}^{2}-\mathrm{N}^{2}$ & 1,538 & 1,660 & & 367,714 & 362,835 \\
$\mathrm{~N}^{2}-\mathrm{P}^{3}$ & 1,603 & 1,740 & & 269,013 & 273,083 \\
$\mathrm{P}^{3}-\mathrm{N}^{3}$ & 1,508 & 1,492 & & 411,140 & 378,749 \\
$\mathrm{~N}^{3}-\mathrm{C}^{2}$ & 1,440 & 1,368 & & 249,796 & 222,711 \\
$\mathrm{Cl}^{1}-\mathrm{P}^{1}$ & 2,008 & 1,937 & & 145,491 & 109,253 \\
$\mathrm{Cl}^{2}-\mathrm{P}^{1}$ & 2,008 & 1,937 & & 145,491 & 109,253 \\
$\mathrm{Cl}^{3}-\mathrm{P}^{2}$ & 2,010 & 2,033 & & 143,889 & 143,085 \\
$\mathrm{Cl}^{4}-\mathrm{P}^{2}$ & 2,010 & 2,033 & & 143,889 & 143,085 \\
$\mathrm{Cl}^{5}-\mathrm{P}^{3}$ & 2,051 & 2,068 & & 117,014 & 113,284 \\
$\mathrm{Cl}^{6}-\mathrm{P}^{3}$ & 2,051 & 2,068 & & 117,014 & 113,284 \\
$\mathrm{H}^{1}-\mathrm{C}^{1}$ & 1,082 & 1,093 & & 243,024 & 243,511 \\
$\mathrm{H}^{2}-\mathrm{C}^{1}$ & 1,083 & 1,093 & & 241,151 & 239,981 \\
$\mathrm{H}^{3}-\mathrm{C}^{1}$ & 1,083 & 1,093 & & 241,151 & 239,981 \\
$\mathrm{H}^{4}-\mathrm{C}^{2}$ & 1,083 & 1,074 & & 236,372 & 235,058 \\
$\mathrm{H}^{5}-\mathrm{C}^{2}$ & 1,088 & 1,076 & 228,429 & 227,102 \\
$\mathrm{H}^{6}-\mathrm{C}^{2}$ & 1,088 & 1,076 & 228,429 & 227,102
\end{tabular}

local minimum of the force field approximation of the PES. This gauche conformation was also found with a $\mathrm{RHF} / 6-31 \mathrm{G}^{*}$ ab initio geometry optimization. However, the ab initio results show that the gauche conformation corresponds to a local minimum of the PES, and the planar trans-cis conformation corresponds to the global minimum of the PES.

An unrestricted geometry optimization of the eclipsed conformation with the force field shows that the eclipsed conformation corresponds to a transition state with respect to the dihedral angle $\mathrm{N}^{1} \mathrm{P}^{2}-\mathrm{N}^{2} \mathrm{P}^{3}$ (the transition state lies between stable conformations corresponding to dihedral angles $\angle \mathrm{N}^{1} \mathrm{P}^{2}-\mathrm{N}^{2} \mathrm{P}^{3}=126,2^{\circ}$ and $\angle \mathrm{N}^{\prime} \mathrm{P}^{2}-\mathrm{N}^{2} \mathrm{P}^{3}=233,8^{\circ}$ ). Ab initio geometry optimizations found no indication of local minima of the PES which correspond to these two conformations. In addition, the ab initio calculations showed that the planar eclipsed conformation corresponds to a minimum of the PES with respect to variations of the $\mathrm{N}^{1} \mathrm{P}^{2}-\mathrm{N}^{2} \mathrm{P}^{3}$ dihedral angle.

An energy expression which only includes the terms (1)-(4) overestimates the torsional flexibility of $\mathrm{P}-\mathrm{N}$ bonds which form an angle with the main chain (i.e., the 'double' bonds). This leads to a de-stabilization of trans conformations and to the prediction of spurious stable conformations.

The introduction of torsional potential terms (5) with $k^{\prime}=0,5 \mathrm{kcal} / \mathrm{mol}$ for these bonds resolves the problems which were discussed above. The gauche conformation corresponds to a local minimum of the PES, and the planar eclipsed conformation 
Tab. 5. Geometry optimized bond angles $\theta_{j}$, zero-strain bond angles $\theta_{j}^{0}$, second derivatives of the PES $H_{j j}$, and constants $k_{j}^{\mathrm{b}}$ for the dichlorophosphazene trimer

\begin{tabular}{|c|c|c|c|c|}
\hline \multirow[t]{2}{*}{ Angle } & $\theta_{j}$ & $\theta_{j}^{0}$ & $H_{j j}$ & $k_{j}^{\mathrm{b}}$ \\
\hline & \multicolumn{2}{|c|}{ in degree } & \multicolumn{2}{|c|}{ in $\mathrm{kcal} \cdot \mathrm{mol}^{-1} \cdot$ degree $^{-2}$} \\
\hline$\angle C^{1} P^{1} N^{1}$ & 110,81 & 114,37 & 0,385 & 0,403 \\
\hline$\angle \mathrm{P}^{\prime} \mathrm{N}^{\prime} \mathrm{P}^{2}$ & 135,34 & 140,05 & 0,050 & 0,530 \\
\hline$\angle N^{1} P^{2} N^{2}$ & 113,37 & 117,99 & 0,501 & 0,544 \\
\hline$\angle \mathrm{P}^{2} \mathrm{~N}^{2} \mathrm{P}^{3}$ & 134,31 & 165,66 & 0,050 & 0,080 \\
\hline$\angle \mathrm{N}^{2} \mathrm{P}^{3} \mathrm{~N}^{3}$ & 115,97 & 118,50 & 0,504 & 0,533 \\
\hline$\angle \mathrm{P}^{3} \mathrm{~N}^{3} \mathrm{C}^{2}$ & 131,61 & 92,89 & 0,105 & 0,033 \\
\hline$\angle \mathrm{Cl}^{1} \mathrm{P}^{1} \mathrm{~N}^{1}$ & 115,19 & 117,90 & 0,413 & 0,343 \\
\hline$\angle \mathrm{Cl}^{2} \mathrm{P}^{1} \mathrm{~N}^{1}$ & 115,19 & 117,90 & 0,413 & 0,343 \\
\hline$\angle \mathrm{Cl}^{3} \mathrm{P}^{2} \mathrm{~N}^{2}$ & 114,54 & 114,60 & 0,425 & 0,420 \\
\hline$\angle \mathrm{Cl}^{4} \mathrm{P}^{2} \mathrm{~N}^{2}$ & 114,54 & 114,60 & 0,425 & 0,420 \\
\hline$\angle C l^{5} \mathrm{P}^{3} \mathrm{~N}^{3}$ & 115,74 & 114,80 & 0,414 & 0,389 \\
\hline$\angle \mathrm{Cl}^{6} \mathrm{P}^{3} \mathrm{~N}^{3}$ & 115,74 & 114,80 & 0,414 & 0,389 \\
\hline$\angle \mathrm{H}^{\prime} \mathrm{C}^{1} \mathrm{~N}^{1}$ & 110,83 & 111,59 & 0,233 & 0,230 \\
\hline$\angle \mathrm{H}^{2} \mathrm{C}^{1} \mathrm{~N}^{1}$ & 108,30 & 109,97 & 0,228 & 0,230 \\
\hline$\angle \mathrm{H}^{3} \mathrm{C}^{1} \mathrm{~N}^{1}$ & 108,30 & 109,97 & 0,228 & 0,230 \\
\hline$\angle \mathrm{H}^{4} \mathrm{C}^{2} \mathrm{~N}^{3}$ & 108,90 & 108,35 & 0,299 & 0,294 \\
\hline$\angle \mathrm{H}^{5} \mathrm{C}^{2} \mathrm{~N}^{3}$ & 112,24 & 110,85 & 0,295 & 0,278 \\
\hline$\angle \mathrm{H}^{6} \mathrm{C}^{2} \mathrm{~N}^{3}$ & 112,24 & 110,85 & 0,295 & 0,278 \\
\hline$\angle \mathrm{Cl}^{1} \mathrm{P}^{1} \mathrm{C}^{1}$ & 119,58 & 119,61 & 0,311 & 0,291 \\
\hline$\angle \mathrm{Cl}^{2} \mathrm{P}^{1} \mathrm{Cl}^{1}$ & 120,84 & 115,58 & 0,314 & 0,217 \\
\hline$\angle \mathrm{Cl}^{3} \mathrm{P}^{2} \mathrm{~N}^{1}$ & 120,83 & 120,83 & 0,352 & 0,355 \\
\hline$\angle \mathrm{Cl}^{4} \mathrm{P}^{2} \mathrm{Cl}^{3}$ & 118,34 & 117,37 & 0,314 & 0,316 \\
\hline$\angle C l^{5} \mathrm{P}^{3} \mathrm{~N}^{2}$ & 122,21 & 122,21 & 0,338 & 0,349 \\
\hline${ }_{2} \mathrm{Cl}^{6} \mathrm{P}^{3} \mathrm{Cl}^{5}$ & 115,59 & 114,42 & 0,339 & 0,345 \\
\hline$\angle \mathrm{H}^{2} \mathrm{C}^{1} \mathrm{H}^{1}$ & 120,85 & 120,22 & 0,195 & 0,193 \\
\hline$\angle \mathrm{H}^{3} \mathrm{C}^{1} \mathrm{H}^{1}$ & 120,85 & 120,22 & 0,195 & 0,193 \\
\hline$\angle \mathrm{H}^{5} \mathrm{C}^{2} \mathrm{H}^{4}$ & 119,09 & 119,67 & 0,205 & 0,205 \\
\hline$\angle \mathrm{H}^{6} \mathrm{C}^{2} \mathrm{H}^{4}$ & 119,09 & 119,67 & 0,205 & 0,205 \\
\hline
\end{tabular}

corresponds to a minimum (instead of a transition state) with respect to torsions around the $\mathrm{P}^{2}-\mathrm{N}^{2}$ bond, if the torsional potential terms are included in the energy expression.

\section{Results and discussion}

The results of a relaxed scan around the $\mathrm{N}^{2}-\mathrm{P}^{3}$ bond with the force field with torsional potential energy terms, and the ab initio molecular orbital calculations are shown in Figs. 7-9. The force field calculations reproduce the torsional potential of the $\mathrm{N}^{2}-\mathrm{P}^{3}$ bond well for torsional motions within $\pm 100^{\circ}$ of the planar trans-cis conformation. The force field over-estimates the torsional barrier by approximately $0,8 \mathrm{kcal} / \mathrm{mol}$, and predicts that the eclipsed conformation is a transition state (with 


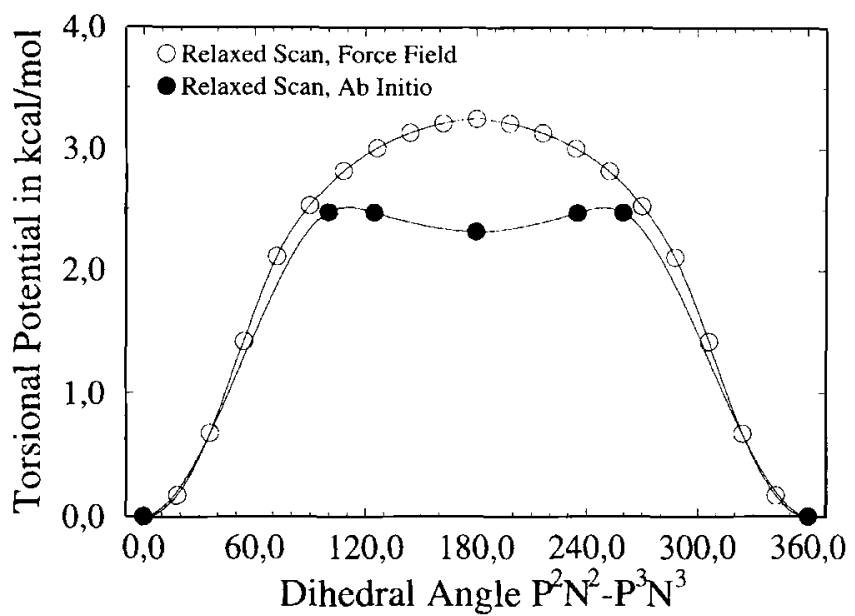

Fig. 7. Relaxed scan around the $\mathrm{N}^{2}-\mathrm{P}^{3}$ bond (angle in degree) with the force field. The filled symbols refer to results of the ab initio molecular orbital calculations

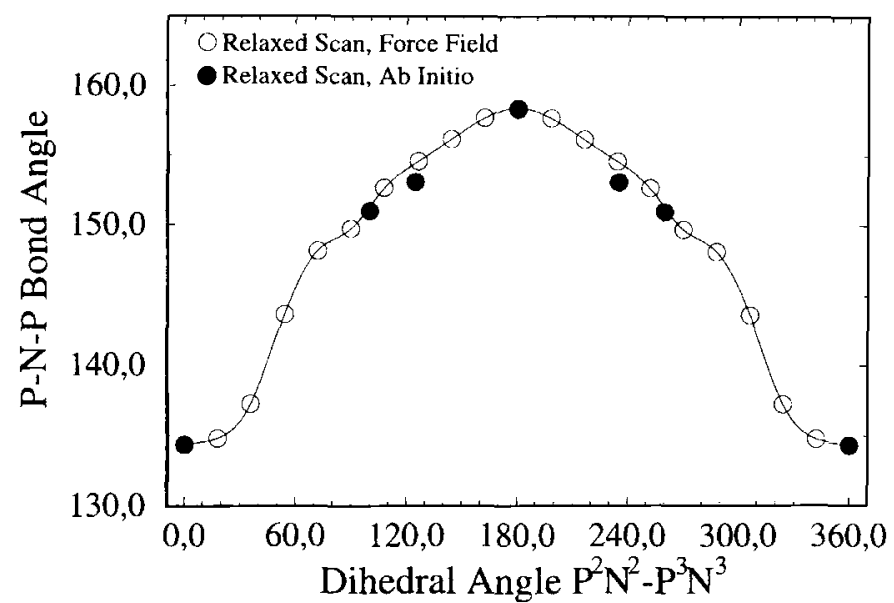

Fig. 8. Change in the $P^{2} N^{2} P^{3}$ bond angle during a relaxed scan around the $N^{2}-P^{3}$ bond with the force field. The filled symbols refer to results of the ab initio molecular orbital calculations. Angles are given in degree

respect to a rotation around the $\mathrm{N}^{2}-\mathrm{P}^{3}$ bond) instead of a local minimum of the PES. The variation of the $P^{2} N^{2} P^{3}$ bond angle and the $N^{1} P^{2}-N^{2} P^{3}$ dihedral angle during a rotation around the $\mathrm{N}^{2}-\mathrm{P}^{3}$ bond are in excellent agreement with the results of the ab initio molecular orbital calculations. 


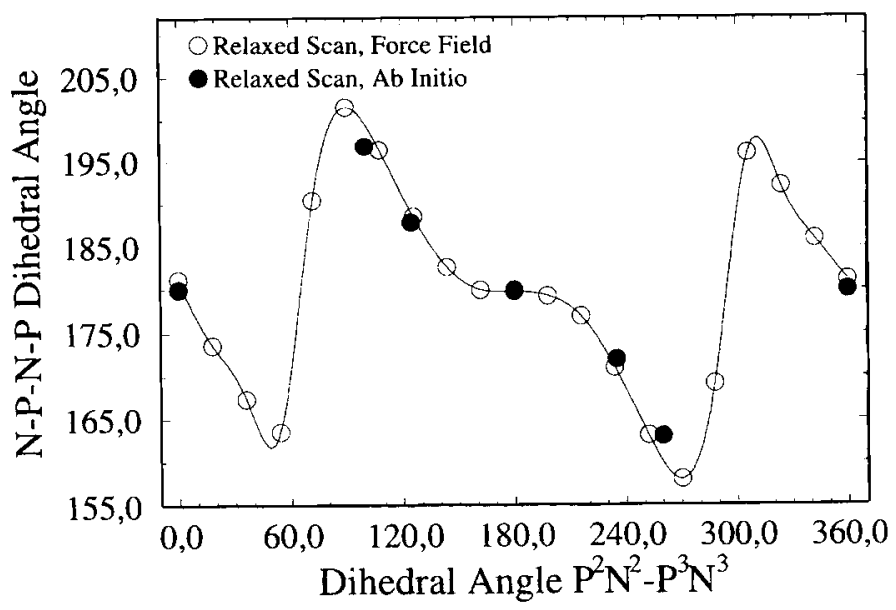

Fig. 9. Change in the $\mathrm{N}^{1} \mathrm{P}^{2}-\mathrm{N}^{2} \mathrm{P}^{3}$ dihedral angle during a relaxed scan around the $\mathrm{N}^{2}-\mathrm{P}^{3}$ bond with the force field. The filled symbols refer to results of the ab initio molecular orbital calculations. Angles are given in degree

Further developments and improvements of the force field should take its future applications into account. The force field which is introduced in the previous section focuses on the ability to reproduce ab initio results on stable conformations and relaxed scans around the central $\mathrm{P}-\mathrm{N}$ bond in a dichlorophosphazene trimer. In order to achieve a good agreement between force field and ab initio results for conformers which form during a rotation, the torsional potential terms were introduced. The torsional potential terms improve the performance of the force field in the vicinity of the conformations which were studied with the ab initio technique. This emphasizes that previous ab initio studies of the regions of the PES which are investigated with the force field are essential for obtaining reliable results with the force field technique. Since the torsional potentials which were studied with the ab initio technique and bond-stretch and angle bend force constants are largely determined by the immediate molecular environment of a bond ${ }^{23)}$, the parameterization for the trimer can be extended to a parameterization for larger molecules.

This force field is well-suited to study main chain motions of larger molecules for which the conformation of a local chain segment remains close to the stable conformation (e.g. one could consider a 'twist' motion of a chain which is created by concerted small variations of dihedral angles) ${ }^{30,31)}$. In addition, it is well-suited for studying motions which involve full rotations around main chain bonds (e.g. crankshaft-type motions) $)^{32,33)}$.

However, one should proceed with caution if this force field is used for conformational studies of molecular geometries which are far away from the conformations which were studied with the ab initio technique. The calculation of conformational entropies can be problematic as it involves extensive scans of the PES ${ }^{4,5}$. 
A first step in order to extend the reliability of the force field is to investigate the physical and/or chemical origins of the torsional potential terms (5). The following approximations which were used to develop the force field can provide an explanation for the torsional potential terms:

(a) The electrostatic interaction of electrons and nuclei is approximated by the electrostatic interaction of nuclear centered point charges (i.e., we only consider the interaction of electrostatic monopoles). A more accurate description of the electrostatic interaction would include higher multipole moments.

(b) Throughout the whole rotation of a part of the molecule around a bond, we use partial atomic charges which were derived from the ground state conformation. We therefore neglect the polarizability of the charge distribution. A rigid rotor scan (RHF/6-31+G*) showed that the NPA partial atomic charges of the substituents vary only slightly (up to $6 \%$ ) during torsional motion. The polarizability of the charge distribution will most likely lead to the variations in higher multipole moments during a rotation. The polarizability of selected regions of the molecular electron density (e.g. the polarizability of an 'electron cloud' in the vicinity of a substituent) can be studied by investigating changes in higher multipole moments during a torsion.

Nuclear centered multipole moments can be determined from ab initio results by the 'distributed multipole analysis' ${ }^{34)}$ or by a least squares fit of the electrostatic potential of the molecule with a multipole expansion ${ }^{35}$.

(c) Previous studies suggest that the bond structure of a $\mathrm{P}-\mathrm{N}$ bond results in no intrinsic barrier to rotation (due to a $\mathrm{p}_{\pi}(\mathrm{N})-\mathrm{d}_{\pi}(\mathrm{P})$ overlap) ${ }^{36)}$. An imperfect $\mathrm{p}_{\pi}(\mathrm{N})$ $\mathrm{d}_{\pi}(\mathrm{P})$ overlap can lead to an intrinsic barrier to rotation around the $\mathrm{P}-\mathrm{N}$ bonds. Although we were able to fit the results of the rigid rotor scan around a single bond entirely with the electrostatic and the van der Waals interaction (see Fig. 6), we can not rule out that the torsional barriers include small contributions from intrinsic barriers to rotation.

The authors thank the Natural Science and Engineering Research Council of Canada (NSERC) and the Ontario Centre for Materials Research (OCMR) for financial support. It is a pleasure to thank Professor Ian Manners for the many helpful discussions, and Ms. Anne Klemperer for the help with the preparation of the manuscript.

1) P. J. Flory, "Statistical mechanics of Chain Molecules", Interscience, New York 1969

2) J. E. Mark, Macromolecules 11, 627 (1978)

3) J. H. Gibbs, E. A. DiMarzio, J. Chem. Phys. 28, 373 (1958)

4) P. R. Sundararajan, Macromolecules 23, 2600 (1990)

5) P. R. Sundararajan, Macromolecules 26, 344 (1993)

6) J. Bicerano, "Computational Modelling of Polymers", Marcel Dekker, Inc., New York 1992

7) B. R. Gelin, "Molecular Modelling of Polymer Structures and Properties", Hanser Publishers, München 1994 
8) G. Trinquier, J. Am. Chem. Soc. 104, 6969 (1982)

9) G. Trinquier, J. Am. Chem. Soc. 108, 568 (1986)

10) K. F. Ferris, P. Friedman, D. M. Friedrich, Int. J. Quantum Chem., Quantum Chem. Symp. 22, 207 (1988)

11) K. F. Ferris, C. B. Duke, Int. J. Quantum Chem., Quantum Chem. Symp. 23, 397 (1989)

12) K. F. Ferris, S. M. Risser, Chem. Phys. Lett. 174, 333 (1990)

13) H. R. Allcock, R. W. Allen, J. J. Meister, Macromolecules 9, 950 (1976)

14) R. W. Allen, H. R. Allcock, Macromolecules 9, 956 (1976)

15) E. Saiz, J. Polym. Sci., Polym. Phys. Ed. 25, 1565 (1987)

16) R. H. Boyd, L. Kesner, J. Am. Chem. Soc. 99, 4248 (1977)

17) M. J. Frisch, G. W. Trucks, M. Head-Gordon, P. M. W. Gill, M. W. Wong, J. B. Foresman, B. G. Johnson, H. B. Schlegel, M. A. Robb, E. S. Replogle, R. Gomperts, J. L. Andreas, K. Raghavachari, J. S. Brinkley, C. Gonzales, R. L. Martin, D. J. Fox, D. J. Defrees, J. Baker, J. J. P. Stewart, J. A. Pople, Gaussian 92, Revision C, Gaussian Inc., Pittsburgh, PA 1992

18) E. D. Glendening, A. E. Reed, J. E. Carpenter, F. Weinhold, NBO version 3.1

19) CERIUS Molecular Modeling Software for Materials Research from Molecular Simulations Inc. of Burlington, MA, and Cambridge, UK

20) M. J. S. Dewar, E. A. C. Lucken, M. A. Whitehead, J. Chem. Soc. 2423 (1960)

21) A. E. Reed, R. B. Weinstock, F. Weinhold, J. Chem. Phys. 82, 735 (1985)

22) D. C. Bott, "Structural Basis for Semiconducting and Metallic Polymers", in "Handbook of Conducting Polymers", vol. 2, ch. 33, T. A. Skotheim, Ed. Marcel Dekker, Inc., New York 1986

23) R. Jaeger, J. B. Lagowski, I. Manners, G. J. Vancso, Macromolecules 28, 539 (1995)

24) E. R. Dawidson, D. Feller, Chem. Rev. 86, 681 (1986)

25) W. J. Welch, "Force-Field Techniques and Their Use in Estimating the Conformational Stability of Polymers", in "Computational Modelling of Polymers", ch. 2, J. Bicerano, Ed. Marcel Dekker, Inc., New York 1992

26) U. Burkert, N. L. Allinger, "Molecular Mechanics", American Chemical Society, Washington, D. C. 1982. ACS Monograph 177

27) T. Clark, "A Handbook of Computational Chemistry", ch. 2, John Wiley \& Sons, New York 1985

$28)$ S. L. Mayo, B. D. Olafson, W. A. Goddard III, J. Phys. Chem. 94, 8897 (1990)

29) W. J. Hehre, L. Radom, P. v. R. Schleyer, J. A. Pople, "Ab Initio Molecular Orbital Theory", John Wiley \& Sons, New York 1986

30) H. Takeuchi, R.-J. Roe, J. Chem. Phys. 94, 7446 (1991)

31) H. Takeuchi, R.-J. Roe, J. Chem. Phys. 94, 7458 (1991)

32) Y. Zhan, W. L. Mattice, Macromolecules 25, 1554 (1992)

33) D. B. Adolf, M. D. Ediger, Macromolecules 25, 1074 (1992)

34) A. J. Stone, M. Alderton, Mol. Phys. 56, 1047 (1985)

35) J. Kong, J.-M. Yang, Int. J. Quantum Chem. 46, 239 (1993)

36) H. R. Allcock, "Phosphorus-Nitrogen Compounds", Academic Press, New York 1972 